\title{
Correction to: Creep and solvent squeeze behavior of k-carrageenan gels under compression
}

\author{
Takuma Tanigawa $^{1} \cdot$ Koichi Yao $^{1} \cdot$ Ryosuke Shimizu $^{1} \cdot$ Jun-ichi Horinaka ${ }^{1} \cdot$ Toshikazu Takigawa $^{1}$
}

Published online: 20 July 2019

(C) Springer-Verlag GmbH Germany, part of Springer Nature 2019

\section{Correction to: Colloid and Polymer Science \\ https://doi.org/10.1007/s00396-019-04526-1}

The author noticed that the published paper contained error. Unfortunately, Table 2 was incorrectly presented. Given in this paper is the correct table.

The original article has been corrected.

Publisher's note Springer Nature remains neutral with regard to jurisdictional claims in published maps and institutional affiliations.

The online version of the original article can be found at https://doi.org/ 10.1007/s00396-019-04526-1

Toshikazu Takigawa

takigawa.toshikazu.7u@kyoto-u.ac.jp

1 Department of Material Chemistry, Kyoto University, Nishikyo-ku, Kyoto 615-8510, Japan 
Table 2 Initial diameter $\left(D_{\text {ini }}\right)$, equilibrium strain of the $i$ th $\operatorname{spring}\left(\varepsilon_{i \text { eq }} ; i=1,2,3\right)$, sum of $\varepsilon_{i \text {,eq }}\left(\sum \varepsilon_{i \text {,eq }}\right)$, spring constant of the $i$ th spring $\left(E_{i} ; i=1,2,3\right)$, and re-defined permeability of the $i$ th cylinder $\left(k_{i}^{\prime} ; i=1,2,3\right)$

\begin{tabular}{llllllllllll}
\hline$D_{\text {ini }}(\mathrm{mm})$ & $\varepsilon_{1, \text { eq }}$ & $\varepsilon_{2, \mathrm{eq}}$ & $\varepsilon_{3, \mathrm{eq}}$ & $\sum \varepsilon_{i \text {,eq }}$ & $\varepsilon_{\mathrm{eq}}$ & $E_{1}(\mathrm{kPa})$ & $E_{2}(\mathrm{kPa})$ & $E_{3}(\mathrm{kPa})$ & $k_{1}^{\prime}\left(10^{-12} \mathrm{~m}^{2} \mathrm{Pas}\right)$ & $k_{2}^{\prime}\left(10^{-12} \mathrm{~m}^{2} \mathrm{Pas}\right)$ & $k_{3}^{\prime}\left(10^{-12} \mathrm{~m}^{2} \mathrm{Pas}\right)$ \\
\hline 10 & 0.15 & 0.37 & 0.18 & 0.70 & 0.74 & 51 & 21 & 43 & 0.086 & 0.85 & 1.7 \\
15 & 0.16 & 0.32 & 0.24 & 0.72 & 0.75 & 50 & 25 & 33 & 0.080 & 0.75 & 2.4 \\
20 & 0.17 & 0.44 & 0.12 & 0.73 & 0.74 & 47 & 18 & 67 & 0.10 & 1.5 & 2.7 \\
25 & 0.083 & 0.33 & 0.25 & 0.66 & 0.75 & 96 & 24 & 32 & 0.053 & 0.71 & 2.4 \\
\hline
\end{tabular}

